\title{
An Improved DC Line Fault Detection Scheme Using Zone Partition for MTDC Wind Power Integration Systems
}

\author{
Saizhao Yang, Wang Xiang, Member, IEEE, Jinyu Wen, Member, IEEE
}

\begin{abstract}
The MMC based DC grids are an effective solution to integrate bulk wind power. Under DC faults, the wind power is continuously fed into DC grids, resulting in large fault currents. To guarantee the uninterrupted and safe operation of healthy parts, DC faults should be detected and isolated selectively. Most existing DC fault detection schemes rely on large current-limiting reactors (CLR) to guarantee high selectivity. The reliability of them will be deteriorated under weak boundary conditions. Besides, some schemes fail to identify close-in faults. Though fault detection schemes independent of CLRs are proposed, they cannot work well for remote faults. Hence, to protect the entire transmission line with smaller CLRs, an improved DC fault detection scheme using zone partition is proposed. Firstly, according to different fault distances, internal faults are partitioned into four zones along the transmission line. The fault characteristics in different zones are analyzed. Then, the polarities and arrival times of traveling-waves are used to design the criteria dedicated to different zones. The proposed method is endurable to fault resistance and noise disturbance. Simulation results show that the MTDC wind power integration systems can operate safely during DC fault isolation.
\end{abstract}

Index Terms - DC fault detection, Zone Partition, HVDC transmission, modular multilevel converter, wind power.

\section{INTRODUCTION}

Large-scale wind power utilization can effectively deal with the issues of fossil fuel shortage and environmental pollution [1][2]. At the end of 2019, the total installed capacity of wind power rises to $651 \mathrm{GW}$ worldwide [3]. Considering the intermittence and fluctuation characteristics of wind power, the modular multilevel converter (MMC) based high-voltage direct current (HVDC) transmission technology has been recognized as a promising solution to integrate bulk wind power. Recently, with the merits of operational flexibility and power supply reliability, the multi-terminal HVDC (MTDC) wind power integration systems have attracted increasing attention [4][5]. For example, the world's first $\pm 500 \mathrm{kV}$ DC grid project, Zhangbei project, transmits $4500 \mathrm{MW}$ wind power to the load center Beijing [6].

For China, Brazil, and some other countries, the onshore wind power integration accounts for a larger proportion [7]. For the onshore wind power integration systems, the overhead lines (OHL) are preferred for large-scale power transmission over long-distance [8]. Being exposed to the air, the OHL has a great likelihood of fault occurrence.

This work is sponsored by the Joint Funds of the National Natural Science Foundation of China (U1766211) and the Fundamental Research Funds for the Central Universities (2021JYCXJJ026). (Corresponding author: Wang Xiang)

W. Xiang is with the Department of Electronic and Electrical Engineering, University of Strathclyde, Glasgow G1 1XW, U.K. (e-mail: xiangwang1003@foxmail.com)

S. Yang, J. Wen are with the State Key Laboratory of Advanced Electromagnetic Engineering and Technology, Huazhong University of Science and Technology, Wuhan 430074, China. (e-mail: saizhaoyang@foxmail.com, jinyu.wen@ hust.edu.cn).
Additionally, due In the event of DC faults, SM capacitors will discharge to the fault point, leading to the rapid drop of the SM capacitor voltage to the continuous wind power transmission, the fault current and the converter's arm current rise rapidly within several milliseconds, which harms the safe operation of the entire system [6]. To avoid overcurrent and breakdown of the entire system, some active current-limiting control and fault-through control schemes are proposed to guarantee safe operation [9]-[11]. For MTDC systems, to not

interrupt the power transmission of healthy parts in DC grids, the faulty part is desired to be isolated before the blocking of the MMCs. Thus, the DC faults should be detected selectively and quickly, thereby issuing the trip signal to the high voltage DC circuit breakers (DCCB) to isolate the faulted lines [6].

Regarding the single-end selective DC line fault detection schemes, most existing schemes rely on the boundary effects provided by the current-limiting reactor (CLR) to discriminate internal and external faults. Based on different fault detection principles, they can also be categorized into the following three groups: 1) The traveling-wave (TW) based methods in time-domain [12]-[16]. 2) The wavelet (WT) or mathematical morphology gradient (MMG) based methods [6][17]. 3) The CLR voltage based methods [18][19]. As pointed out in [20], for boundary protection schemes, large CLRs (more than $100 \mathrm{mH}$ ) are required to guarantee high reliability and selectivity. Various simulation results demonstrate that $200 \mathrm{mH}$ CLRs are required to guarantee robustness to high fault resistances and noise disturbance [21]. However, the cost and weight of CLRs should also be considered, which means the sizes of CLR may not be sufficient for selective and reliable protection design. For instance, in existing MMC-HVDC projects, the maximum size of CLRs that can provide the boundary effects is $150 \mathrm{mH}$ [22]. Besides, there are several scenarios where the configured CLRs are small that cannot provide strong boundary conditions, including the two-terminal HVDC systems and fault tolerable converter based HVDC systems. For example, $50 \mathrm{mH}$ and $100 \mathrm{mH}$ CLRs are respectively selected for INELFE two-terminal MMC-HVDC project [23] and Kunliulong three-terminal HVDC project constructed by hybrid MMC [24]. Besides, among these aforementioned schemes, the methods in [14]-[16] cannot identify close-in faults correctly.

Pilot protection schemes can guarantee high reliability to identify external and internal faults under weak boundary conditions [25][26]. However, they are highly dependent on the communication between stations, which is sensitive to data code error and synchronization error [21]. Hence, single-end non-boundary fault detection schemes independent of CLRs are required. In [27], the arrival time difference between line-mode and zero-mode TWs is adopted to achieve the non-boundary fault detection. This scheme is robust to high-resistance faults. However, it cannot identify pole-to-pole 
(PTP) faults. To improve the applicability for different fault types, TW frequency characteristics which are dependent on the fault distances are adopted to identify internal faults in [28]. Whereas, these non-boundary schemes cannot work well for remote faults. Except for [27] [28], there are no other references concerning single-end fault detection schemes that do not rely on CLRs.

To be concluded, existing DC fault detection schemes are restricted by the sizes of CLRs. In addition, only using some simple travelling-wave detection methods, such as detecting the arrival times or polarities of the first and second backward traveling-waves, there exist protection dead-zones for close-in faults and remote faults [14]-[16].To address the challenges, an improved DC fault detection scheme using zone partition is proposed for the MTDC wind power integration system to achieve uninterrupted and safe operation. Considering the high-impedance faults are difficult to be identified, the high-impedance fault detection is the top concern of this paper. Based on different zone partitions, the fault analysis is conducted. Then, the polarities and arrival times of the backward TWs are adopted to detect internal and external faults. As shown in Fig. 1, the proposed method can protect the entire transmission line in the case of small CLRs. In addition, it is endurable to fault resistances and noise disturbance.

The rest of the paper is organized as follows. Section II depicts the topology and the layout of the MTDC wind power integration system. The protected transmission line is partitioned into four zones and the fault TW behaviors in different zones are analyzed in section III. Based on the analysis, section III proposes the DC fault detection criteria dedicated to different zones. The robustness and reliability of the proposed protection scheme are verified by simulations in section V. Finally, conclusions are drawn in section VI.

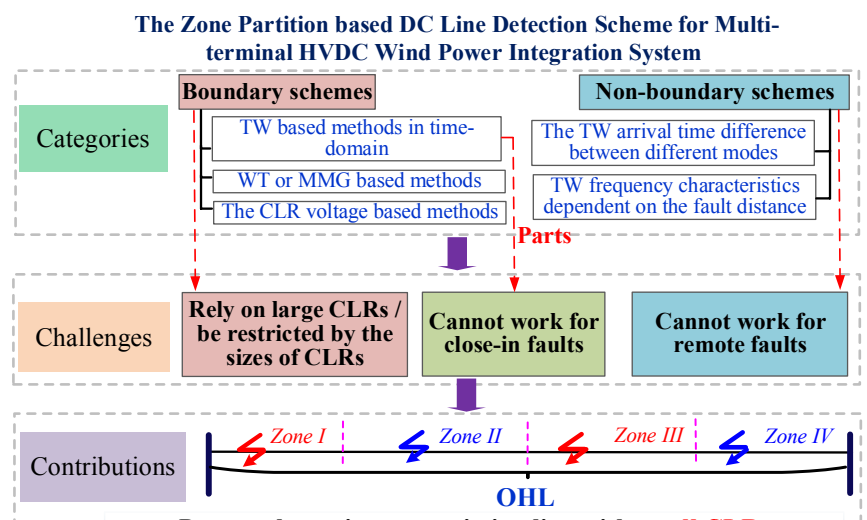

Protect the entire transmission line with small CLRs.

Fig. 1. The challenges of existing schemes and the contributions of the proposed scheme.

\section{DC FAULT TW BEHAVIOUR ANALYSIS IN DIFFERENT ZONES}

\section{A. The Zone Partitions of the Transmission Line}

To protect the entire transmission line, particularly for close-in faults and remote faults, internal faults are partitioned into four zones, thereby designing fault detection criteria dedicated to different zones. Taking the relay $\mathrm{CB} 12$ as an example, based on different fault distances, the zone partition of the transmission line is illustrated in Fig. 2. Where close-in faults and remote faults are located in Zone I and Zone IV respectively.
Since the minimum or maximum identifiable fault distance is affected by the sampling frequency greatly, there is no explicit range to define the zone boundaries. In conventional AC power system protection, the protective range of the distance protection stage $I$ is $80 \%-95 \%$ of the entire transmission line. As the sampling frequency improves, for the high-precision distance protection or TW protection, the dead-zone will shrink. For example, in [28], when the sampling step is selected as $500 \mathrm{~ns}$, the protection dead-zone is only $300 \mathrm{~m}$.

Based on the aforementioned analysis, considering that the sampling frequency is selected as $6 \mu$ s, Zone $I$ is ranging from 0 to $5 \%$ of the transmission line and Zone $I V$ is ranging from $95 \%$ to $100 \%$. Zone II is ranging from $5 \%$ to $50 \%$ and Zone $I I I$ is ranging from $50 \%$ to $95 \%$. Since a margin is considered during zone partitions, the internal faults in Zone I (IV) may be identified by the criterion of Zone II (III). However, it will not cause false fault identification results.

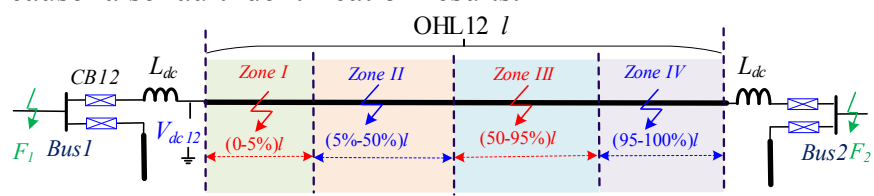

Fig. 2. The diagram of DC faults under different zones.

\section{B. TW Reflections and Refractions at Different Locations}

As shown in Fig. 3, under DC faults, the negative initial backward TW $V_{i}$ induced from the fault point travels towards the terminals of OHL (points $A$ and $B$ ). The initial TW is reflected at points $A$ and $B$ respectively. The reflected TWs $V_{A B}$ and $V_{B \beta}$ can be calculated as:

$$
V_{A(B) \beta}=\beta_{A(B)} V_{i}
$$

where $\beta_{A(B)}$ is the reflection coefficient of point $A(B)$.

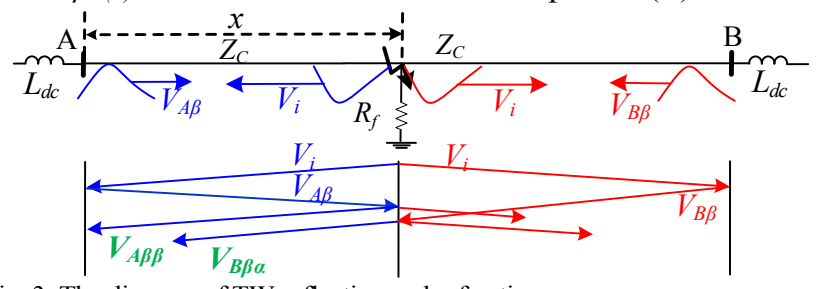

Fig. 3. The diagram of TW reflection and refraction.

The reflected TWs $V_{A(B) \beta}$ travel towards the fault point and are reflected at the fault point again. The reflected TW $V_{A(B) \beta \beta}$ and refracted TW $V_{A(B) \beta \alpha}$ can be calculated as:

$$
V_{A(B) \beta \beta}=\beta_{F} V_{A(B) \beta} ; V_{A(B) \beta \alpha}=\alpha_{F} V_{A(B) \beta}
$$

where $\beta_{F}$ and $\alpha_{F}$ are the reflection and refraction coefficients at the fault point respectively.

\section{1) The Reflections and Refractions at the Terminal of $\mathrm{OHL}$}

Based on the Peterson law [14], the reflection and refraction at point $A$ can be analyzed using the following equivalent circuit, as shown in Fig. 4:

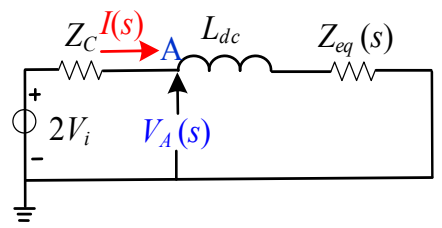

Fig. 4.The Peterson equivalent circuit in s domain.

Based on the equivalent circuit, it can be obtained: 


$$
I(s)=\frac{2 V_{i}}{Z_{C}+Z_{e q}(s)+s L_{d c}}
$$

where $Z_{C}$ is the wave impedance of the OHL and $L_{d c}$ is the current-limiting reactance. $Z_{e q}$ is the equivalent impedance of the converter, which can be expressed as:

$$
Z_{e q}=s L_{e q}+R_{e q}+\frac{1}{s C_{e q}}=\frac{2 s L_{0}}{3}+\frac{2 R_{0}}{3}+\frac{N}{6 s C_{0}}
$$

where $L_{0}, R_{0}$ and $C_{0}$ are the arm inductance, arm resistance and sub-module capacitance respectively. $N$ is the number of the sub-module per arm. For meshed DC grids, considering multiple parallel lines connected to the same DC busbar, the equivalent impedance of parallel lines connected to the same DC busbar should also be accounted into $Z_{e q}$.

Further, voltage $V_{A}(s)$ can be obtained as:

$$
V_{A}(s)=2 V_{i}-I(s) Z_{C}=\frac{Z_{e q}(s)+s L_{d c}}{Z_{C}+Z_{e q}(s)+s L_{d c}} \times 2 V_{i}
$$

Then, the reflected TW $V_{A \beta}$ and reflection coefficient $\beta_{A(B)}$ can be calculated as:

$$
V_{A \beta}=V_{A}(s)-V_{i}=\beta_{A(B)} V_{i}=\left\lfloor 1-\frac{2 Z_{C}}{Z_{C}+Z_{e q}(s)+s L_{d c}}\right\rfloor \times V_{i}
$$

Equation (6) shows that the reflected wave $V_{A \beta}$ is smoothed by CLRs and the reflection coefficient $\beta_{A(B)}$ is time-varying. Using the initial value theorem, at the initial constant of the arrival of the initial TW $V_{i}$, the reflection coefficient $\beta_{A(B)}$ can be obtained as:

$$
\beta_{A(B)}(t=0)=\lim _{s \rightarrow \infty} \beta_{A(B)}(s)=1
$$

Equation (7) shows the reflection at the terminal of OHL will not change the polarities of the wave-fronts of TWs.

2) The Reflection and Refraction at the Fault Point

The reflection coefficient $\beta_{F}$ and refraction coefficient $\alpha_{F}$ can be calculated as:

$$
\beta_{F}=\frac{R_{f} / / Z_{C}-Z_{C}}{R_{f} / / Z_{C}+Z_{C}} ; \alpha_{F}=\frac{2 R_{f} / / Z_{C}}{R_{f} / / Z_{C}+Z_{C}}
$$

Apparently, the reflection coefficient $\beta_{F}$ is smaller than zero. To be concluded, the polarities of wave-fronts and the amplitudes of TWs can be obtained in Table 1 .

Table 1 The polarities of wave-front and the amplitudes of TWs

\begin{tabular}{c|c|c||c|c|c}
\hline TW & Amplitudes & $\begin{array}{c}\text { polarities of } \\
\text { wave-fronts }\end{array}$ & TW & Amplitudes & $\begin{array}{c}\text { polarities of } \\
\text { wave-fronts }\end{array}$ \\
\hline$V_{i}$ & $V_{i}$ & Negative & $V_{A \beta \beta}$ & $\beta_{F} \beta_{A} V_{i}$ & Positive \\
\hline$V_{A(B) \beta}$ & $\beta_{A} V_{i}$ & Negative & $V_{B \beta \alpha}$ & $\alpha_{F} \beta_{A} V_{i}$ & Negative \\
\hline
\end{tabular}

Equation (8) also demonstrates that the amplitude of the refracted wave $V_{B \beta a}$ is determined by the fault resistance $R_{f}$. When the fault resistance is small, the refracted wave $V_{B \beta \alpha}$ can be negligible. Particularly, for solid faults, the refracted wave $V_{B \beta \alpha}$ is equal to zero. Thus, in the case of low-resistance faults, for the measured point $A$, the refracted wave $V_{B \beta \alpha}$ will not be detected. With the fault resistance increasing, the amplitude of the refracted wave $V_{B \beta \alpha}$ increases. For the measured point $A$, the arrival times of the TWs $V_{A \beta \beta}$ and $V_{B \beta \alpha}\left(t_{a}\left(V_{A \beta \beta}\right)\right.$ and $\left.t_{a}\left(V_{B \beta \alpha}\right)\right)$ can be calculated as:

$$
t_{a}\left(V_{A \beta \beta}\right)=\frac{3 x}{v} ; t_{a}\left(V_{B \beta \alpha}\right)=\frac{2 l-x}{v}
$$

where $v$ is the TW propagation speed and $x$ is the fault distance.
As can be seen in (9), the arrival times of the TWs $V_{A \beta \beta}$ and $V_{B \beta \alpha}$ are dependent on the fault distances. Thus, the following analysis is conducted based on different zones.

\section{Fault Analysis of Different Zones}

Assuming that the direction from the MMC to the OHL is positive, the TWs $V_{i}, V_{A \beta \beta}$ and $V_{B \beta \alpha}$ are all backward TWs. For the relay CB12, the busbar fault $F_{l}$ is the backward external fault while $F_{2}$ is the forward external fault. The backward external fault $F_{l}$ can be sorted out by the directional element easily. Thus, the solid bus fault $F_{2}$ is the worst external case and it is difficult to be identified.

1) Fault Analysis in Zone III

Based on equation (9), in the case of faults in Zone III, the refracted wave $V_{B \beta a}$ will arrive at the point $A$ earlier than the reflected wave $V_{A \beta \beta}$. Since the TW $V_{B \beta \alpha}$ is negative, the backward TW $V_{b}$ will decrease abruptly once TW $V_{B \beta \alpha}$ arrives. The time interval when the TWs $V_{i}$ and $V_{B \beta \alpha}$ arrive at point $A$ $\left(\Delta t\left(V_{i}\right.\right.$ and $\left.\left.V_{B \beta \alpha}\right)\right)$ can be calculated as:

$$
\Delta t\left(V_{i} \text { and } V_{B \beta \alpha}\right)=\frac{2(l-x)}{v}
$$

Subsequently, the reflected wave $V_{A \beta \beta}$ arrives at point $A$, leading to the rapid rise of the backward TW $V_{b}$. Hence, the detected backward TW $V_{b}$ at point $A$ can be obtained, as shown in Fig. 5.

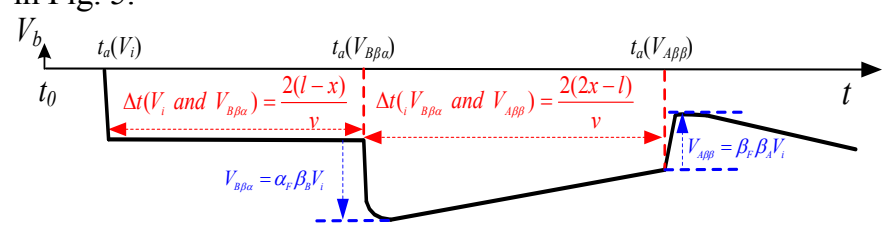

Fig. 5. The detected $V_{b}$ at point $A$ in the case of faults in Zone III.

2) Fault Analysis in Zone II

In the case of faults in Zone II, the reflected wave $V_{A \beta \beta}$ will arrive at point $A$ earlier. Thus, after the arrival of the initial TW $V_{i}$, the backward TW $V_{b}$ rises rapidly before the abrupt drop. The time interval when TWs $V_{i}$ and $V_{A \beta \beta}$ arrive $\left(\Delta t\left(V_{i}\right.\right.$ and $\left.\left.V_{A \beta \beta}\right)\right)$ can be calculated as:

$$
\Delta t\left(V_{i} \text { and } V_{A \beta \beta}\right)=\frac{2 x}{v}
$$

The detected backward TW $V_{b}$ at point $A$ is presented in Fig. 6 .

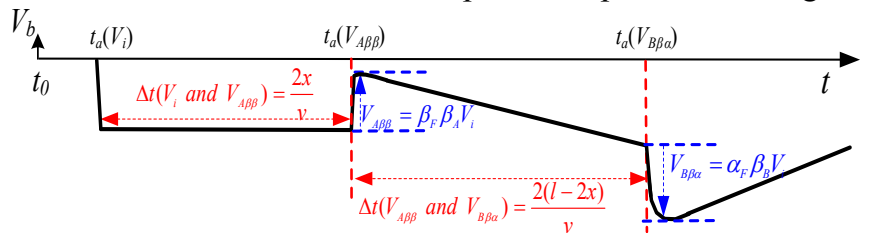

Fig. 6. The detected $V_{b}$ at point $A$ in the case of faults in Zone II.

3) Fault Analysis in Forward Bus Fault $F_{2}$

Under the forward bus fault $F_{2}$, the initial negative TW $V_{i}$ travels towards point $A$ through the current-limiting reactors, as shown in Fig. 7. The refracted TW $V_{i \alpha}$ can be obtained as:

$$
V_{i \alpha}=\alpha_{B} V_{i}
$$

where $\alpha_{B}$ is the refraction coefficient at point $B$. Since CLR will not change the polarities of the wave-fronts of TWs, the wave-front of the refracted wave $V_{i \alpha}$ is also negative. Since the reflection coefficients at points $A$ and $B$ are both positive, the wave-fronts of the detected backward TWs $V_{A \beta \beta}$ and $V_{A \beta \beta \beta \beta}$ at point $A$ are both negative. The time intervals $\Delta t\left(V_{i \alpha}\right.$ and $\left.V_{A \beta \beta}\right)$ 
and $\Delta t\left(V_{i \alpha}\right.$ and $\left.V_{A \beta \beta \beta \beta}\right)$ are:

$$
\Delta t\left(V_{i \alpha} \text { and } V_{A \beta \beta}\right)=\frac{2 l}{v} ; \Delta t\left(V_{i \alpha} \text { and } V_{A \beta \beta \beta \beta}\right)=\frac{4 l}{v}
$$

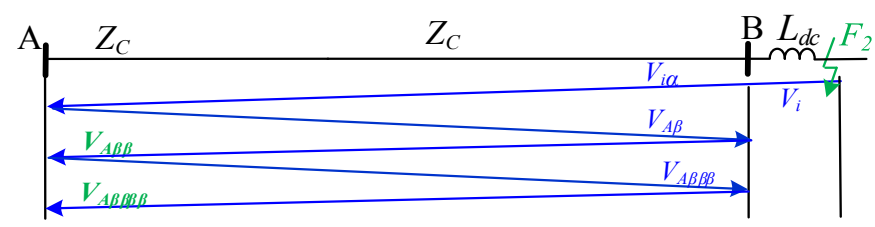

Fig. 7. The TW reflections and refractions under the busbar fault $F_{2}$.

4) Fault Analysis in Zone I

\section{a) The Challenges of Close-in Fault Detection}

Under close-in faults, there exist multiple reflections between the fault point and point $A$ in a short time, as shown in Fig. 8. Due to the short time interval, several subsequent backward TWs cannot be captured accurately. Particularly, when the DC fault occurs at point $A$, the reflected wave $V_{A \beta B}$ cannot be detected.

Considering a large time interval $\Delta t\left(V_{i}\right.$ and $\left.V_{B \beta \alpha}\right)$, the reflected wave $V_{B \beta \alpha}$ may provide a potential approach to identify close-in faults. However, the detection of the reflected wave $V_{B \beta \alpha}$ will arise a new challenge. The detailed analysis is as follows.

As can be seen in Table 1, the polarity of the wave-front of the TW $V_{B \beta \alpha}$ is negative and the time interval $\Delta t\left(V_{i}\right.$ and $\left.V_{B \beta \alpha}\right)$ is close to $2 l / v$. Whereas, under the bus fault $F_{2}$, the detected second backward TW $V_{A \beta \beta}$ is also negative and the time intervals $\Delta t\left(V_{i \alpha}\right.$ and $\left.V_{A \beta \beta}\right)$ is equal to $2 l / v$. Thus, the reflected wave $V_{B \beta \alpha}$ cannot be adopted to detect close-in faults.

\section{b) Difference of Close-in Faults and the Fault $F_{2}$}

Based on the aforementioned analysis, in the case of the busbar fault $F_{2}$, the third detected backward TW $V_{A \beta \beta \beta \beta}$ is negative. Whereas, in the case of close-in faults, the third detected backward TW is $V_{B \beta \beta \beta} \alpha$, which can be calculated as:

$$
V_{B \beta \beta \beta \alpha}=\alpha_{B} \beta_{F}\left(\beta_{B}\right)^{2} V_{i}
$$

Equation (14) shows that the wave-front of the TW $V_{B \beta \beta \beta \alpha}$ is positive. Hence, the wave-front polarities of the third detected backward TW can be adopted to identify close-in faults.

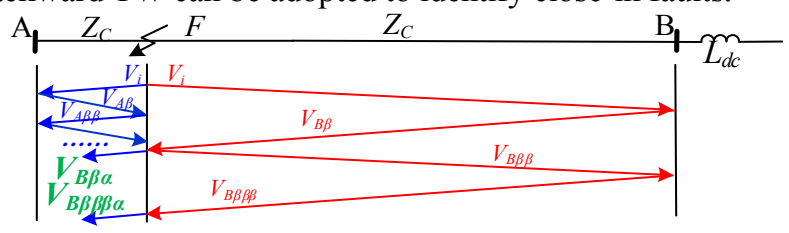

Fig. 8. The TW reflections and refractions in the case of Zone I

\section{5) Fault Analysis in Zone IV}

The TW reflections and refractions in Zone $I V$ are shown in Fig. 9. As can be seen in equation (10), under remote faults, due to the small time interval $\Delta t\left(V_{i}\right.$ and $\left.V_{B \beta \alpha}\right)$, the refracted wave $V_{B \beta \alpha}$ cannot be detected accurately, which proposes challenges on the remote fault detection.

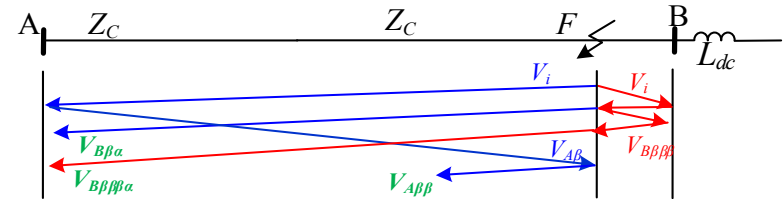

Fig. 9. The TW reflections and refractions in the case of Zone $I V$.

Regarding the fault analysis in Zone $I V$, it is conducted based on two following cases.
Case I: The refracted TW $V_{B \beta \beta \beta \alpha}$ can be detected, which results in a sharp rise of the backward TW. The time interval $\Delta t$ $\left(V_{i}\right.$ and $\left.V_{B \beta \beta \beta \alpha}\right)$ is

$$
\Delta t\left(V_{i} \text { and } V_{B \beta \beta \beta \alpha}\right)=\frac{4 l-3 x}{v}
$$

Case II: The refracted TW $V_{B \beta \beta \beta \alpha}$ cannot be detected. Based on the aforementioned analysis, under the bus fault $F_{2}$, the wave-front of the second detected backward TW $V_{A \beta \beta}$ is negative. Whereas, under remote faults, the wave-front of the second detected backward TW $V_{A \beta \beta}$ is positive.

Regardless of cases I and II, the wave-front polarities of the second detected backward TW can be adopted to identify remote faults correctly.

To be concluded, the detected second and third backward TWs under different zones can be obtained, as shown in Table 2. As can be seen, for internal faults, except Zone III, positive backward TWs can be detected.

Within Zone III, the maximum time interval $\max \left[\Delta t\left(V_{i}\right.\right.$ and $\left.\left.V_{B \beta \alpha}\right)\right]$ is:

$$
\max \left[\Delta t\left(V_{i} \text { and } V_{B \beta \alpha}\right)\right]=\left.\frac{2(l-x)}{v}\right|_{x=0.5 l}=\frac{l}{v}
$$

As can be seen from equation (16), the time interval between the initial backward TW and the second backward TW under Zone III is smaller than that under the external fault $F_{2}$.

\begin{tabular}{|c|c|c|c|}
\hline \multicolumn{2}{|c|}{ Zone } & The second backward TW & The third backward TW \\
\hline \multicolumn{2}{|c|}{ Zone I } & $V_{B \beta \alpha}$ (negative) & $V_{B \beta \beta \beta \alpha}$ (positive) \\
\hline \multicolumn{2}{|c|}{ Zone II } & $V_{A \beta B}$ (positive) & 1 \\
\hline \multicolumn{2}{|c|}{ Zone III } & $\begin{array}{c}V_{B \beta \alpha}(\text { negative }) \\
\max \left[\Delta t\left(V_{i} \text { and } V_{B \beta \alpha}\right)\right]=l / v\end{array}$ & l \\
\hline \multirow{2}{*}{$\begin{array}{l}\text { Zone } \\
I V\end{array}$} & Case I & $V_{B \beta \beta \beta \alpha}$ (positive) & 1 \\
\hline & \begin{tabular}{|l|} 
Case II \\
\end{tabular} & $V_{A \beta \beta}$ (positive) & 1 \\
\hline \multicolumn{2}{|c|}{ Bus fault $F_{2}$} & $\begin{array}{c}V_{A \beta \beta}(\text { negative }) \\
\Delta t\left(V_{i \alpha} \text { and } V_{A \beta \beta}\right)=2 l / v\end{array}$ & $V_{A \beta \beta \beta \beta}$ (negative) \\
\hline
\end{tabular}

Thus, the polarities of backward TWs together with their arrival times can be adopted to identify internal and external faults.

\section{The DC FAult DeteCtion CRiterion Design DEDICATED TO DIFFERENT ZONES}

\section{A. The Mathematical Morphology Gradient and MM Filter \\ 1) The Mathematical Morphology Gradient (MMG)}

To detect the arrival of backward TWs, the mathematical morphology gradient (MMG) is adopted to design the fault detection criterion. For the MMG technology, the upper and lower edges of the signal $f$ are extracted by using two structure elements $g+$ and $g$ - at different origin positions respectively as:

$$
\left\{\begin{array}{l}
\rho^{+}(n)=\left(f \oplus g^{+}\right)(n)+\left(f \Theta g^{+}\right)(n) \\
\rho^{-}(n)=\left(f \Theta g^{-}\right)(n)+\left(f \oplus g^{-}\right)(n) \\
M M G(n)=\rho^{-}(n)-\rho^{+}(n)
\end{array}\right.
$$

where structure elements $g^{+}$and $g$ - are respectively as:

$$
g^{+}=\left\{g_{1}, g_{2}, \ldots, g_{l-1}, \underline{g_{l}}\right\} ; g^{-}=\left\{\underline{g_{1}}, g_{2}, \ldots, g_{l-1}, g_{l}\right\}
$$

where $l$ is the length of the structure element and the underscore represents the origin position.

In (17), “ $\oplus$ " and " $\Theta$ " represent the erosion and dilatation operations. The erosion and dilatation operations of the structure element $g$ on the signal $f$ are expressed as: 


$$
\left\{\begin{array}{l}
(f \oplus g)(x)=\max _{y}\{f(x-y)+g(y)\} \\
(f \Theta g)(x)=\min _{y}\{f(x+y)-g(y)\}
\end{array}\right.
$$

\section{2) The Mathematical Morphology Filter (MMF)}

To avoid noise disturbance, the MMF based on the opening-closing (OC) operation has been adopted. The opening operation "." and closing operation "o" are:

$$
(f \circ g)(n)=(f \Theta g \oplus g)(n) ;(f \bullet g)(n)=(f \oplus g \Theta g)(n)(20)
$$

The opening operation can be adopted to weaken the noise at the signal peak while the closing operation can be used to weaken the noise at the signal trough. To suppress the noise better, the alternate hybrid filter (AHF) is adopted.

$$
\left\{\begin{array}{c}
A H F=0.5 \times\left[F_{O C}(f(x))+F_{C O}(f(x))\right] \\
F_{O C}(f(x))=((f \circ g) \bullet g)(x) \\
F_{C O}(f(x))=((f \circ g) \circ g)(x)
\end{array}\right.
$$

\section{B. Criterion Design Dedicated to Different Zones}

\section{1) The Protection Criterion I}

Assuming that the MMG of the initial backward TW is positive. For Zone II or the case I of Zone IV, the detected second backward TW $V_{A \beta \beta}$ or $V_{B \beta \beta \beta \alpha}$ is positive. Thus, the MMG of the second backward TW is negative, as shown in Fig. 10 (a)(b). Besides, the maximum time intervals $\max \left[\Delta t\left(V_{i}\right.\right.$ and $\left.\left.V_{A \beta \beta}\right)\right]$ and $\max \left[\Delta t\left(V_{i}\right.\right.$ and $\left.\left.V_{B \beta \beta \beta \alpha}\right)\right]$ are respectively as:

$$
\left\{\begin{array}{c}
\max \left[\Delta t\left(V_{i} \text { and } V_{A \beta \beta}\right)\right]=2 x /\left.v\right|_{x=0.5 l}=l / v \\
\max \left[\Delta t\left(V_{i} \text { and } V_{B \beta \beta \beta \alpha}\right)\right]=4(l-x) /\left.v\right|_{x=0.95 l}=0.2 \mathrm{l} / \mathrm{v}
\end{array}\right.
$$

For Zone III, as can be seen from (16), the MMGs of the second backward TW are within $l / v$, as shown in Fig. 10(c).

To be concluded, if the second MMG is detected within the time interval $l / v$, the fault is identified to be internal. Considering a margin for the time interval $\Delta t$, the fault detection criterion I for Zones II, III and the case I of Zone IV is:

$$
|M M G|>0.5 \text { and } \Delta t \leq 1.25 \mathrm{l} / \mathrm{v}
$$

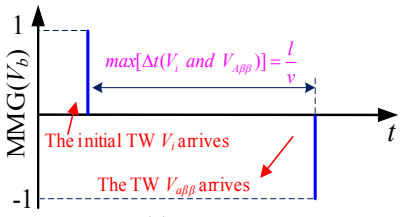

(a) Zone II

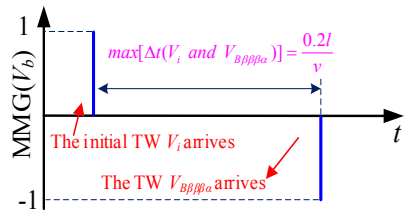

(b) Case $I$ of Zone $I V$

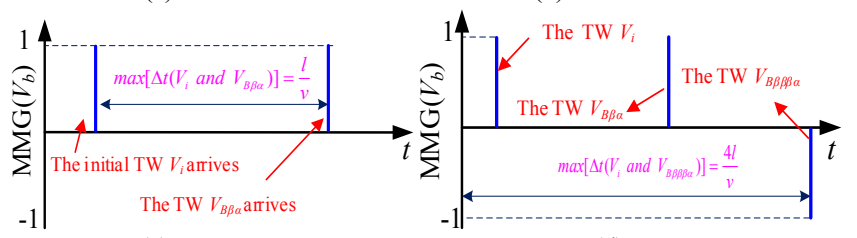

(c) Zone III

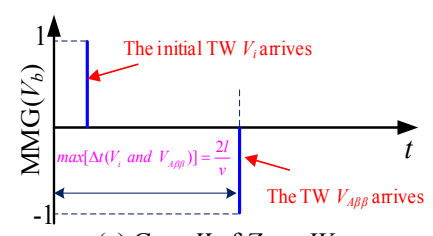

(e) Case II of Zone IV

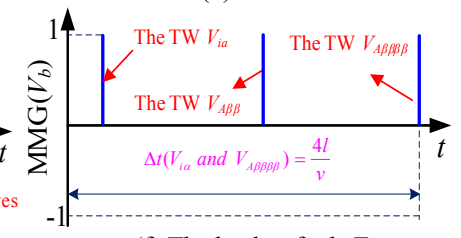

(f) The busbar fault $F_{2}$
Fig. 10. The diagram of the MMG under different zones.

2) The Protection Criterion II

For Zone I, the case II of Zone IV, and the busbar fault $F_{2}$, the diagrams of MMG under these Zones can be obtained, as shown in Fig. 10 (d)-(f). For the bus fault $F_{2}$, the detected MMGs are all positive. Whereas, in the event of Zone I and the case II of Zone IV, negative MMGs can be detected. In addition, the maximum time intervals $\max \left[\Delta t\left(V_{i}\right.\right.$ and $\left.\left.V_{B \beta \beta \beta \alpha}\right)\right]$ and $\max \left[\Delta t\left(V_{i}\right.\right.$ and $\left.\left.V_{A \beta \beta}\right)\right]$ are:

$$
\left\{\begin{array}{c}
\max \left[\Delta t\left(V_{i} \text { and } V_{B \beta \beta \beta \alpha}\right)\right]=4(l-x) /\left.v\right|_{x=0}=4 l / v \\
\max \left[\Delta t\left(V_{i} \text { and } V_{A \beta \beta \alpha}\right)\right]=2 x /\left.v\right|_{x=l}=2 l / v
\end{array}\right.
$$

To be concluded, if a negative MMG is detected within the time interval $4 l / v$, the fault is identified to be internal. Considering margins for the amplitude of $M M G$ and time interval $\Delta t$ respectively, the fault detection criterion II for Zone $I$ and the case II of Zone $I V$ is:

$$
M M G<-0.5 \text { and } \Delta t \leq 4.25 \mathrm{l} / \mathrm{v}
$$
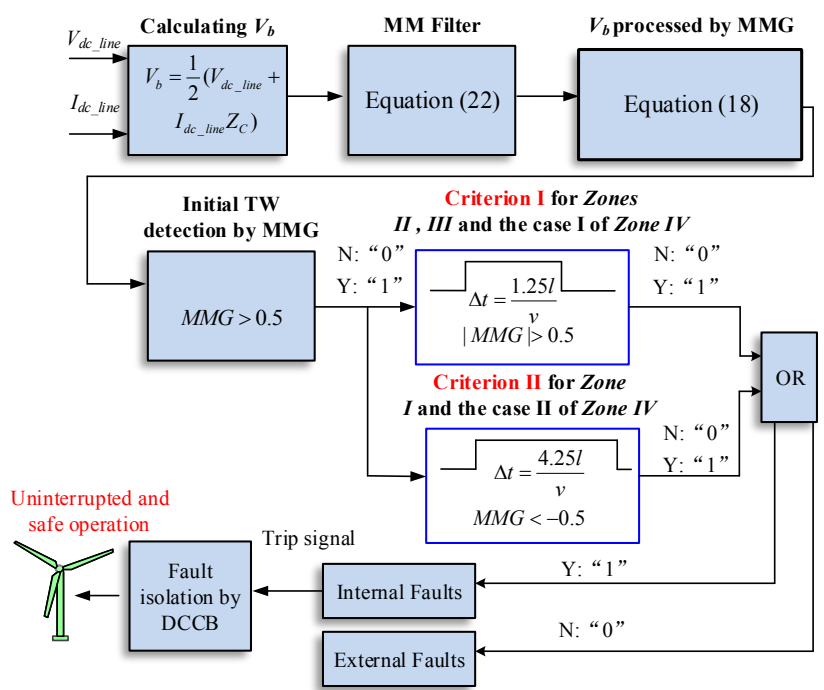

Fig. 11. The flowchart of the overall DC fault detection scheme.

Thus, the overall DC fault detection scheme for the uninterrupted and safe operation of the MTDC wind power integration system can be obtained, as shown in Fig. 11.

\section{THE TOPOLOGY AND LAYOUT OF THE MTDC WIND POWER INTEGRATION SYSTEM}

The topology of the MTDC wind power integration system is shown in Fig. 12. Each wind farm consists of a direct-drive permanent magnet synchronous generators (PMSG), a generator side VSC (GS VSC) and an integration side VSC (IS VSC).

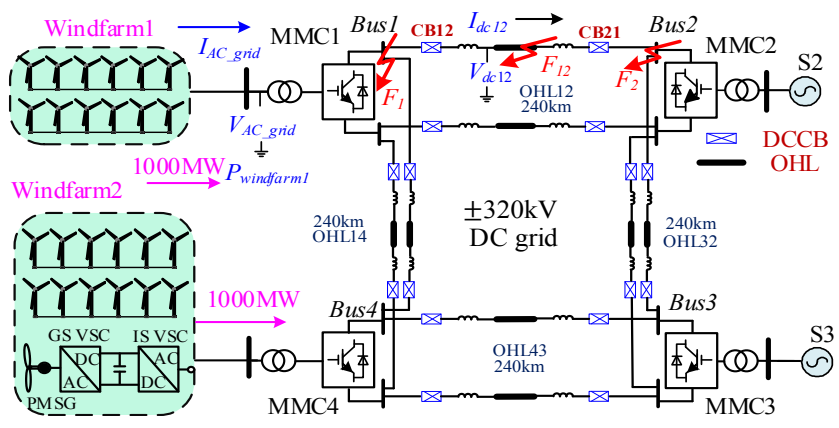

Fig. 12. The topology of the MTDC wind power integration system. 


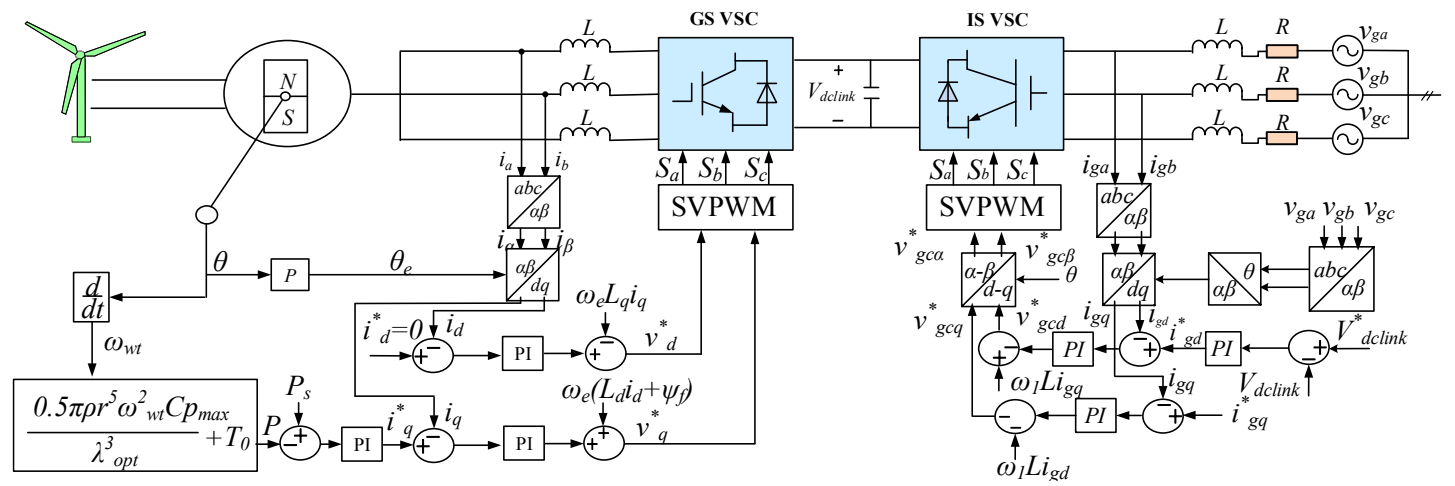

Fig. 13. The controller diagram of the wind farm.

For the wind turbine, the maximum power point tracking (MPPT) algorithm is adopted to obtain the active power reference. The converter GS VSC regulates the back-to-back converters' DC-link voltage by controlling the generator currents via a field-oriented control technique [29], where pitch angle control, torque control, and pitch compensation control prompt the angular frequency $\omega_{m}$ to be coincident with the reference value $\omega_{\text {ref }}$. For the converter IS VSC, the constant DC voltage control is adopted to balance the power flow [30], as shown in Fig. 13.

In meshed $\mathrm{DC}$ grids, $\mathrm{MMC} 3$ controls the $\mathrm{DC}$ voltage while MMC2 controls the active power. For converters MMC1 and MMC4 connected with the wind farms, the grid forming control is adopted to regulate its frequency and $\mathrm{AC}$ voltage [31]. To suppress the fault current rise rate, CLRs are installed at the terminals of OHLs. The hybrid DCCBs with the fast interruption speed and the low on-state loss are adopted to isolate the DC faults.

\section{V.SiMULATION VALIDATIONS}

The MTDC wind power integration system shown in Fig. 12 is built in the PSCAD/EMTDC platform. Various fault cases are simulated to verify the feasibility of the fault detection scheme. The current-limiting reactor is selected as $0.1 \mathrm{H}$ and the overhead line adopts the frequency-dependent model. The system is a symmetric monopole structure. The AC transformer adopts the $\mathrm{Y} / \mathrm{Y}$ connection and the secondary side that is close to the converter is grounded by a large resistance. The structure is robust to pole-to-ground faults [18]. Thus, pole-to-pole faults are the main concern in this paper. The other converter parameters are listed in Table 3.

Table 3 Parameters of each converter station

\begin{tabular}{c||cccc}
\hline Converter & MMC1 & MMC2 & MMC3 & MMC4 \\
\hline arm inductance $/ \mathrm{mH}$ & 50 & 50 & 50 & 50 \\
$\mathrm{SM}$ capacitor $/ \mathrm{mF}$ & 4 & 4 & 8 & 8 \\
$\mathrm{SM}$ number $/ N$ & 200 & 200 & 200 & 200 \\
rated power $/ \mathrm{MW}$ & 750 & 750 & 1500 & 1500 \\
\hline
\end{tabular}

Compared with the zero-mode backward TW $V_{b 0}$, the line-mode backward TW $V_{b l}$ has a steady propagation speed [27]. Thus, the line-mode backward TW $V_{b l}$ is adopted to identify the internal and external faults. Besides, taking the relay $\mathrm{CB} 12$ as an example, the simulations are conducted. Considering the line length $l$ is $240 \mathrm{~km}$ and the TW propagation speed is close to the light speed, the detection times $\Delta t$ in criteria and (25) are respectively $1 \mathrm{~ms}$ and $3.4 \mathrm{~ms}$.

\section{A. Simulation Analysis of Close-in Faults}

DC fault with $150 \Omega$ fault resistance is applied in Zone $I$ and the fault distance is $3 \mathrm{~km}$. The line-mode backward TW $V_{b l}$ and MMG are shown in Fig. 14 (e) and (f) respectively.

As shown in Fig. 14(e), in the case of Zone I, due to the small fault distance, there exist multiple refractions and reflections in a short time interval. Thus, after the initial TW, the following TWs including the TW $V_{A \beta \beta}$ are difficult to be detected. Subsequently, the TWs $V_{B \beta \alpha}$ with the negative wave-front and $V_{B \beta \beta \beta a}$ with the positive wave-front arrive successively, which brings about the abrupt drop and rise of the backward TW respectively. In Fig. 14(f), the negative MMG is detected within $3.4 \mathrm{~ms}$. Hence, it is deemed to be internal and DCCB12 will be tripped. During fault isolation, the arm-bridge currents $I_{\text {arm }}$ of $\mathrm{MMC1}$ are smaller than twice the rated current $(1.8 \mathrm{kA})$, as shown in Fig. 14(d). Hence, there is no risk of overcurrent.

Under the DC fault, the AC voltage of the windfarm1 connected to the DC grid $V_{A C}$ grid and the transmission active power of the windfarm $1 P_{\text {windfarm }}$ will decrease slightly. Since the fault isolation is fast and the healthy parts of the DC grid can still transmit the wind power, the voltage $V_{A C}$ grid and the power $P_{\text {windfarm I }}$ can restore quickly, as shown in Fig. 14(a) and (b). In addition, the output AC currents of the windfarm $1 I_{A C \text { grid }}$ will not increase significantly, as shown in Fig. 14(c).

To be concluded, during DC fault isolation, the MTDC wind power integration system can still operate uninterruptedly and safely.

\section{B. Simulation Analysis of Zones II and III Faults}

At $2.0 \mathrm{~s}$, DC faults with $150 \Omega$ resistance are applied at $25 \%$ and $75 \%$ of OHL respectively. The line-mode backward TWs $V_{b 1}$ are shown in Fig. 17 (a) and Fig. 18 (a) respectively. As shown in Fig. 17 (a), in the case of Zone II, the initial negative backward TW $V_{i}$ arrives at the terminal of OHL, leading to the rapid drop of the backward TW. The subsequent backward TWs $V_{A \beta \beta}$ and $V_{B \beta \alpha}$ arrive successively, which bring about the abrupt rise and fall of the backward TW respectively. These rapid changes of TW $V_{b l}$ are detected by the MMG, as shown in Fig. 17 (b). The second MMG is detected within $1 \mathrm{~ms}$, which holds criterion (23). Thus, the fault is deemed to be internal and the trip signal is issued to the responding DCCBs.

Within Zone III, the detected second MMG is positive, as shown in Fig. 18 (a). Since the second MMG is detected within $1 \mathrm{~ms}$, the fault is also identified to be internal. 


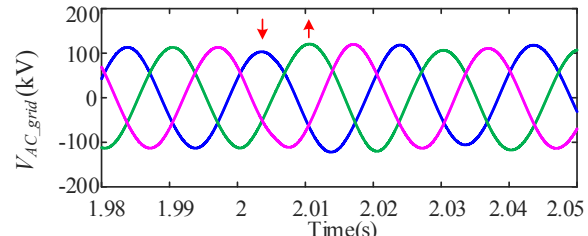

(a) The AC voltage of the windfarm1

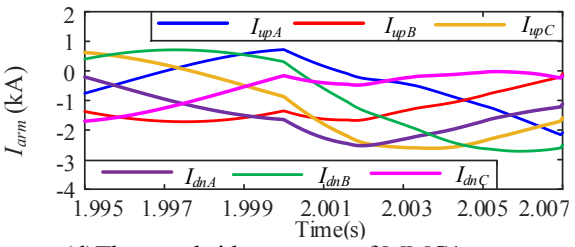

(d)The arm-bridge current of MMC1

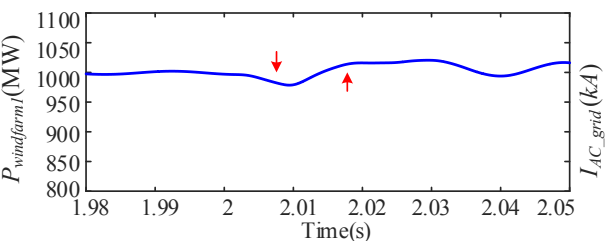

(b)The transmission active power of the windfarm1

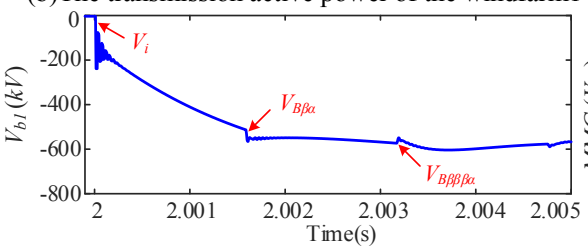

(e)The backward TW $V_{b 1}$

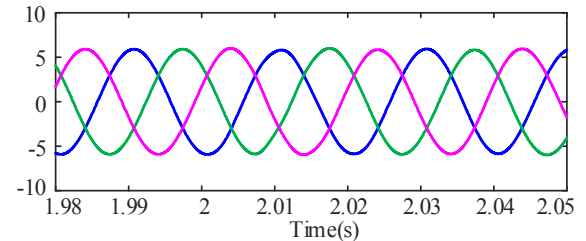

(c) The output AC current of the windfarm1

Fig. 14. The measured backward TWs and MMG under Zone I faults.

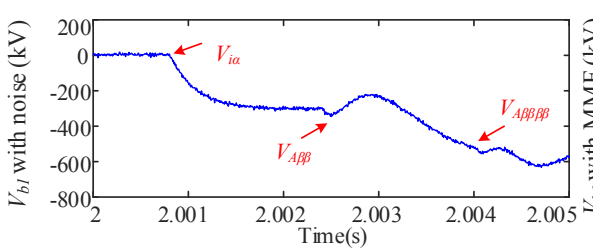

(a)The backward TW $V_{b 1}$ with $40 \mathrm{db}$ noise

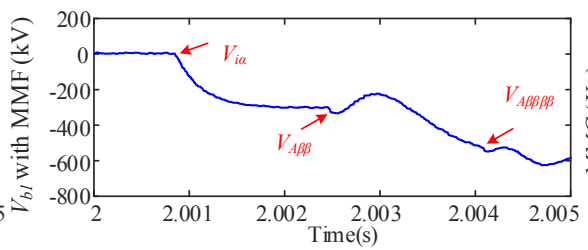

(b) The backward TW $V_{b l}$ with MMF

Fig. 15. The measured backward TWs and MMG in the case of a solid fault $F_{2}$ with $40 \mathrm{~dB}$ noise.

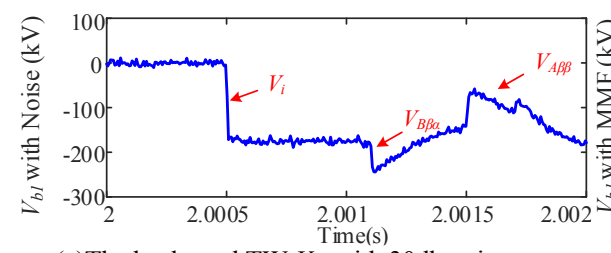

(a)The backward TW $V_{b l}$ with $30 \mathrm{db}$ noise

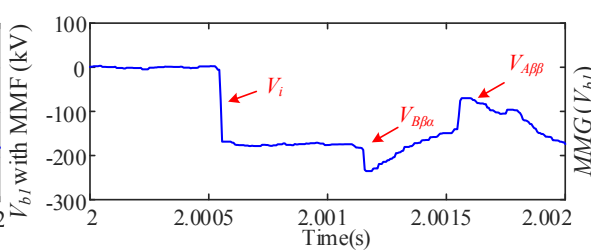

(b) The backward TW $V_{b 1}$ with MMF

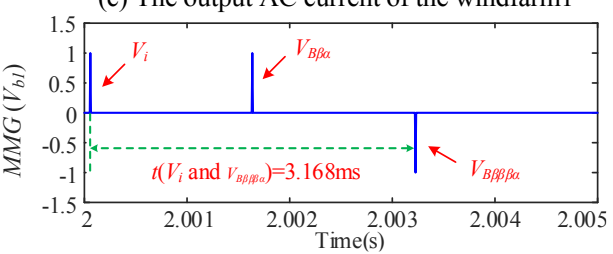

(f)The detected MMG

Fig. 16. The measured backward TWs and MMG in the case of an internal fault with $150 \Omega$ fault resistance and $30 \mathrm{~dB}$ noise.

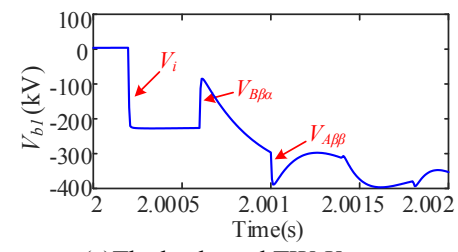

(a) The backward TW $V_{b 1}$

ig. 17. The measured backward TW and MMG in the case of Zone II faults.

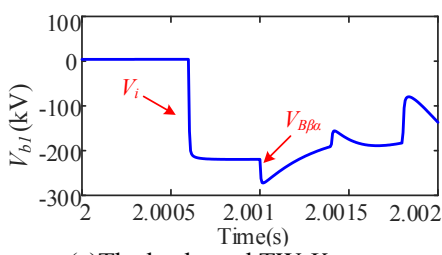

(a)The backward TW $V_{b I}$

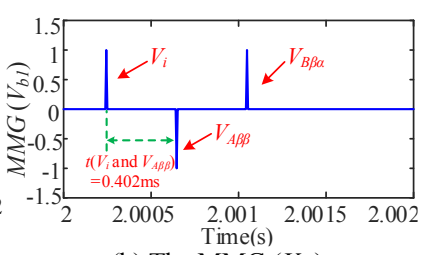

(b) The MMG $\left(V_{b l}\right)$
Fig. 18. The measured backward TW and MMG in the case of Zone III faults.

\section{Simulation Analysis of Remote Faults}

A DC fault with $150 \Omega$ resistance is applied in Zone IV and the fault distance is $237 \mathrm{~km}$. The measured line-mode backward TW $V_{b l}$ and MMG are shown in Fig. 19.

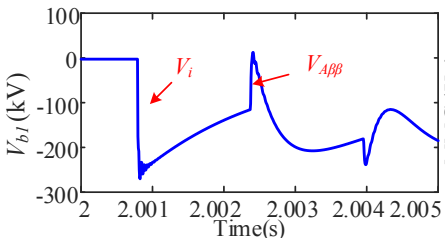

(a)The backward TW $V_{b 1}$

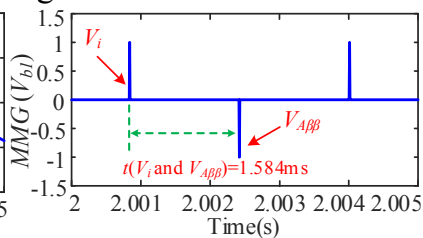

(b) The MMG $\left(V_{b 1}\right)$
Fig. 19. The measured backward TW and MMG in the case of Zone IV.

As shown in Fig. 19 (a), in the case of Zone $I V$ faults, due to the small time interval between the arrivals of the TWs $V_{i}$ and $V_{B \beta \alpha}$, the TW $V_{B \beta \alpha}$ is difficult to be detected. However, the TW $V_{A \beta \beta}$ can be captured accurately. In Fig. 19 (b), the negative MMG is detected within $3.4 \mathrm{~ms}$, which holds the criterion (25). Hence, it is deemed to be an internal fault.

\section{Simulation Analysis of External Fault $F_{2}$}

A solid DC bus bar fault $F_{2}$ is applied, which is regarded as the most severe external fault to test the selectivity of the proposed method. The measured line-mode backward TWs $V_{b 1}$ and MMG are shown in Fig. 20(a) and (b) respectively.

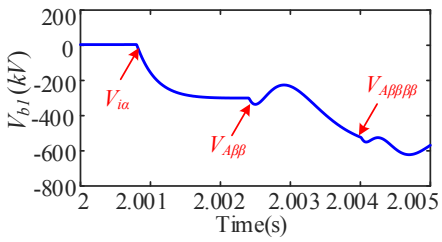

(a)The backward TW $V_{b l}$

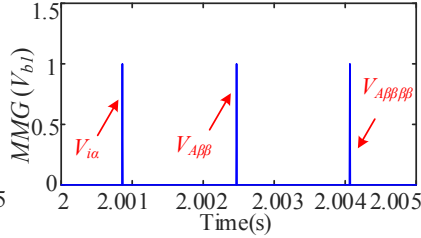

(b) The detected MMG
Fig. 20. The backward TW and MMG in the case of an external fault $F_{2}$

Unlike the detected backward TWs in the case of Zone I and IV faults, the wave-fronts of the detected backward TWs are all positive, as shown in Fig. 20(a). Thus, in Fig. 20(b), no negative MMGs are detected within $3.4 \mathrm{~ms}$. Hence, the fault is identified to be external and no trip signals are delivered to DCCB12.

\section{E. Robustness Analysis of Fault Resistances}

Various internal faults with $500 \Omega$ fault resistance are applied in different zones. The detection times of the proposed scheme under these internal DC faults are shown in Fig. 21.

For Zones II, III, and the case I of Zone $I V$, the faults are 
identified to be internal when the second $\mathrm{MMG}$ is detected within $1 \mathrm{~ms}$. According to equations (10)(11) and (15), in the case of Zone II faults, the larger the fault distance, the longer the detection time of the second MMG. On the contrary, for Zone $I I I$ and the case I of Zone IV faults, the larger the fault distance, the shorter the detection time, as shown in Fig. 21.

For Zone I and the case II of Zone $I V$ faults, they are identified to be internal when the negative $M M G$ is detected within $3.4 \mathrm{~ms}$. The larger the fault distance, the shorter the detection time of the negative MMGs for Zone I while the longer detection time for the case II of Zone IV.

To be concluded, the negative MMGs under these faults can be detected within $3.4 \mathrm{~ms}$ or the second MMG can be detected within $1 \mathrm{~ms}$. Thus, these faults can be identified to be internal correctly. The fault resistance that the proposed method can endure is as high as $500 \Omega$.

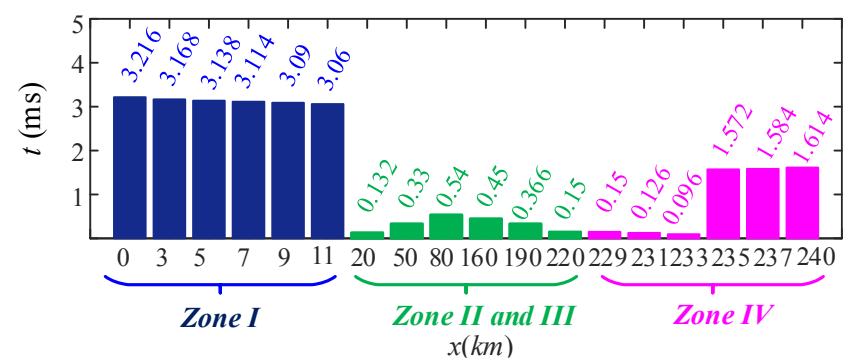

Fig. 21. The detection time under internal faults with $500 \Omega$ fault resistance.

\section{F. Impact Analysis against Noise Disturbance}

A solid external fault $F_{2}$ with $40 \mathrm{~dB}$ noise disturbance and an internal fault with $30 \mathrm{~dB}$ noise disturbance are applied respectively to test the impacts of noise disturbance. Where the fault distance is selected to be $150 \mathrm{~km}$ and the fault resistance is selected to be $300 \Omega$ for the internal fault.

The measured backward TWs $V_{b l}$ with the noise disturbance are shown in Fig. 15(a) and Fig. 16(a) respectively. The noise disturbance can be weakened effectively by the MMF, as shown in Fig. 15(b) and Fig. 16(b). Under the fault $F_{2}$, no negative MMGs are detected, as shown in Fig. 15(c). Hence, the fault is deemed to be external. In the case of Zone III fault, a second MMG is detected within 1ms, as shown in Fig. 16(c). Hence, it is identified to be internal. Thus, the proposed method is endurable to noise disturbance.

\section{G.Discussion on Different CLR Sizes}

CLRs are usually employed to differentiate internal and external faults as the line boundaries. Here, smaller CLRs $(30 \mathrm{mH}$ and $50 \mathrm{mH})$ are adopted to test the robustness of the proposed method under weak boundary conditions. The measured backward TWs and MMGs under the solid external fault $F_{2}$ are shown in Fig. 22 and Fig. 23 respectively.

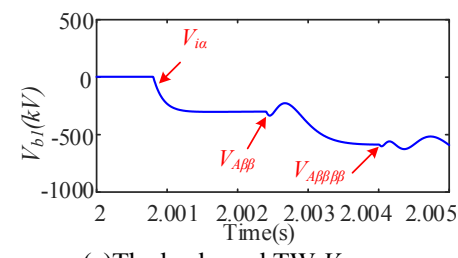

(a)The backward TW $V_{b l}$

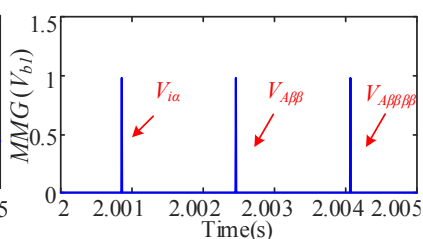

(b) The detected MMG
Fig. 22. The measured backward TW and MMG in the case of 50mH CLR.

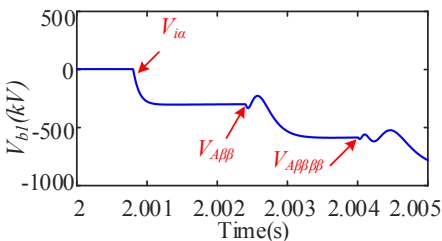

(a) The backward TW $V_{b l}$

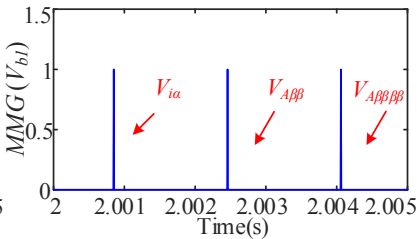

(b) The detected MMG
Fig. 23. The measured backward TW and MMG in the case of 30mH CLR

As can been seen, when the size of CLR decreases, the wave-front of the initial backward TW will be sharper. Whereas, the polarities of the wave-fronts of backward TWs remain unchanged. Hence, there are still no negative MMGs to be detected, which will not trigger the protection falsely. Thus, the proposed method can still work well under weak boundary conditions.

\section{H. Relationship between the Protection Time and the Transmission Line Length}

For Zones II, III, and the case $I$ of Zone $I V$, the faults are identified to be internal when the second MMG is detected within $1 \mathrm{~ms}$. Thus, the detection time of the faults in Zones II, III and the case $I$ of Zone $I V$ can be calculated as:

$$
\left\{\begin{array}{c}
t_{d}(\text { Zone } I I)=\Delta t+\frac{x}{v}=\frac{3 x}{v} \\
t_{d}(\text { Zone } I I I)=\Delta t+\frac{x}{v}=\frac{2 l-x}{v} \\
t_{d}(\text { Case I of Zone } I V)=\Delta t+\frac{x}{v}=\frac{4 l-3 x}{v}
\end{array}\right.
$$

For Zone $I$ and the case $I I$ of Zone $I V$, the faults are identified to be internal when the negative $M M G$ is detected within $3.4 \mathrm{~ms}$. The detection time of the faults in Zone I and the case II of Zone $I V$ can be calculated as:

$$
\left\{\begin{array}{c}
t_{d}(\text { Zone } I)=\Delta t+\frac{x}{v}=\frac{4 l-3 x}{v} \\
t_{d}(\text { Case II of Zone IV })=\Delta t+\frac{x}{v}=\frac{3 x}{v}
\end{array}\right.
$$

As pointed out earlier, high-impedance fault detection is the top concern in this paper. The fault currents under high-impedance faults are much smaller than those under low-impedance faults. Thus, the need for fast detection speed is not the main concern in the manuscript.

Taking the Zhangbei project as an example, the most severe fault is allowed to be detected within $3 \mathrm{~ms}$. While, the longest fault detection time for the proposed method is no more than $3.5 \mathrm{~ms}$. Considering that the transmission line in the simulation test system is longer than the lines in Zhangbei project and these faults are high-impedance ones, the detection speed of the proposed method is fast enough.

\section{CONCLUSION}

To guarantee uninterrupted and safe operation of the MTDC wind power integration systems, an improved DC fault detection scheme using zone partition is proposed. Based on different zones, the polarities and arrival times of the backward TWs are analyzed. Then, the MMG is adopted to detect the arrival of backward TWs, thereby designing the fault detection criteria dedicated to different zones. When the second MMG is detected within $1 \mathrm{~ms}$ or the negative MMG is detected within 
$3.4 \mathrm{~ms}$, it is deemed to be an internal fault. The DCCBs will be tripped quickly to isolate the DC fault.

Compared with some existing time-domain TW-based methods, the proposed fault detection scheme can identify close-in faults correctly. Compared with existing non-boundary protection schemes, the proposed scheme can detect remote faults effectively. In the case of small CLRs, the wave-front polarities and the arrival times of backward TWs remain unchanged. Thus, the proposed method can still work well under weak boundary conditions. To be concluded, the proposed method can protect the entire transmission line with small CLRs. In addition, it is robust to fault resistances and endurable to noise disturbance.

\section{REFERENCES}

[1] A. Moawwad, M. S. El Moursi, W. Xiao, "A Novel Transient Control Strategy for VSC-HVDC Connecting Offshore Wind Power Plant," IEEE Trans. Sustain. Energy, vol. 5, no. 4, pp. 1056-1069, 2014.

[2] W. Xiang, S. Yang, G. P. Adam, et al, "DC Fault Protection Algorithms of MMC HVDC Grids: Fault analysis, Methodologies, Experimental Validations and Future Trends," IEEE Trans. Power Electron., early access, to be published.

[3] "GWEC Global Wind Report 2019". Available at https://gwec.net/globalwind-report-2019/, accessed 2020.

[4] Y. Wang, C. Wang, L. Xu, et al, "Adjustable Inertial Response from the Converter with Adaptive Droop Control in DC Grids," IEEE Trans. Smart Grid, vol. 10, no. 3, pp. 3198-3209, May 2019.

[5] D. Jovcic, M. Taherbaneh, J. Taisne, et al, "Offshore DC Grids as an Interconnection of Radial Systems: Protection and Control Aspects," IEEE Trans. Smart Grid, vol. 6, no. 2, pp. 903-910, March 2015.

[6] W. Xiang, S. Yang, L. Xu, et al., "A Transient Voltage based DC Fault Line Protection Scheme for MMC based DC Grid Embedding DC Breakers," IEEE Trans. Power Del., vol. 34, no. 1, pp. 334-345, 2019.

[7] S. Cao, W. Xiang, X. Lu, et al., "Energy dissipation of MMC-HVDC based onshore wind power integration system with FB-DBS and DCCB," IET Renew. Power Gener., vol. 14, no. 2, pp. 222-230, 2020.

[8] Y. Wen, C. Y. Chung, Z. Shuai, et al., "Towards Flexible Risk-Limiting Operation of Multi-Terminal HVDC Grids with Vast Wind Generation," IEEE Trans. Sustain. Energy, early access, to be published.

[9] D. Jovcic, M. Taherbaneh, J. Taisne, et al., "Offshore DC Grids as an Interconnection of Radial Systems: Protection and Control Aspects," IEEE Trans. Smart Grid, vol. 6, no. 2, pp. 903-910, March 2015.

[10] W. Sanusi, M. Al Hosani, M. S. E. Moursi, "A Novel DC Fault Ride-Through Scheme for MTDC Networks Connecting Large-Scale Wind Parks," IEEE Trans. Sustain. Energy, vol. 8, no. 3, pp. 1086-1095, July 2017.

[11] L. Shi, G. P. Adam, R. Li, et al., "Enhanced Control of Offshore Wind Farms Connected to MTDC Network Using Partially Selective DC Fault Protection," IEEE J. of Emerg. and Sel. Topics in Power Electron., early access, to be published.

[12] W. Leterme, J. Beerten, D. V. Hertem, "Nonunit protection of HVDC grids with inductive DC cable termination", IEEE Trans. Power Del., vol. 31, pp. 820-828, 2016.

[13] J. Sneath, A. Rajapakse, "Fault detection and Interruption in an earthed HVDC grid using ROCOV and hybrid DC breakers," IEEE Trans. Power Del., vol.31, no.3, pp. 973-981, 2016.

[14] C. Zhang, G. Song, T. Wang, et al., "Non-unit Traveling Wave Protection of HVDC Grids Using Levenberg-Marquart Optimal Approximation," IEEE Trans. Power Del., early access, to be published.

[15] C. Zhang, G. Song, X. Dong, "Non-unit Ultra-high-speed DC Line Protection Method for HVDC Grids using First Peak Time of Voltage," IEEE Trans. Power Del., early access, to be published.

[16] Q. Huang, G. Zou, X. Wei, et al., "A non-unit line protection scheme for MMC-based multi-terminal HVDC grid," Int. J. Elect. Power Energy Syst., vol. 107, pp. 1-9, 2019.

[17] L. Tang, X. Dong, S. Shi, et al., "A High-speed Protection Scheme for the DC Transmission Line of a MMC-HVDC Grid," Elect. Power Syst. Research, vol. 168, pp. 81-91, 2019.

[18] R. Li, L. Xu, L. Yao, "DC Fault Detection and Location in Meshed Multi-terminal HVDC Systems based on DC Reactor Voltage Change
Rate,” IEEE Trans. Power Del., vol.32, no.3, pp. 1516-1626, Jun. 2017.

[19] S. Yang, W. Xiang, R. Li, et al., "An Improved DC fault Protection Algorithm for MMC HVDC Grids based on Modal Domain Analysis," IEEE J. of Emerg. and Sel. Topics in Power Electron., early access, to be published.

[20] D. Jovcic, W. Lin, S. Nguefeu, et al., "Low-Energy Protection System for DC Grids Based on Full-Bridge MMC Converters," IEEE Trans. Power Del., vol. 33, no. 4, pp. 1934-1943, 2018.

[21] S. Yang, W. Xiang, M. Zhou, et al., "A Single-end Protection Scheme for Hybrid MMC HVDC Grids Considering the Impacts of the Active Fault Current-limiting Control," IEEE Trans. Power Del., early access, to be published.

[22] S. Yang, W. Xiang, J. Wen, "Analysis of Impact of Grounding-pole Current-limiting Reactor on Fault Detection of MMC based DC Grids," presented in $8^{\text {th }}$ Renewable Power Generation Conference (RPG 2019), Shanghai, China, Oct. 2019, pp. 1-6.

[23] M. A. Abdel-Moamen, S. A. Shaaban, F. Jurado, "France-Spain HVDC Transmission System with Hybrid Modular Multilevel Converter and Alternate-arm Converter," 2017 Innovations in Power and Advanced Computing Technologies (i-PACT), Vellore, 2017, pp. 1-6.

[24] X. Chen, H. Li, Y. Liang, et al., "A protection scheme for hybrid multi-terminal HVDC networks utilizing a time-domain transient voltage based on fault-blocking converters," Int. J. Elect. Power Energy Syst., vol. 118, pp. 105825, 2020.

[25] K. Jia, Z. Xuan, T. Feng, et al, "Transient High-Frequency Impedance Comparison-Based Protection for Flexible DC Distribution Systems," IEEE Trans. Smart Grid, vol. 11, no. 1, pp. 323-333, Jan. 2020.

[26] K. Jia, C. Wang, T. Bi, et al, "Transient Current Correlation based Protection for DC Distribution System," IEEE Trans. Ind. Electron., early access, to be published.

[27] N. Tong, X. Lin, Y. Li, et al., "Local Measurement-Based Ultra-High-Speed Main Protection for Long Distance VSC-MTDC," IEEE Trans. Power Del., vol. 34, no. 1, pp. 353-364, 2019.

[28] S. Yang, W. Xiang and J. Wen, "An Improved DC Fault Protection Scheme Independent of Boundary Components for MMC based HVDC Grids," IEEE Trans. Power Del., early access, to be published.

[29] R. Blasco-Gimenez, S. Añó-Villalba, J. Rodriguez-D'Derlée, "Diode-based HVdc link for the connection of large offshore wind farms," IEEE Trans. Energy Convers., vol. 26, no. 2, pp. 615-626, 2011.

[30] H. Geng, L. Liu, R. Li, "Synchronization and Reactive Current Support of PMSG-Based Wind Farm During Severe Grid Fault," IEEE Trans. Sustain. Energy, vol. 9, no. 4, pp. 1596-1604, 2018.

[31] E. Belenguer, R. Vidal, H. Beltran, et al., "Control strategy for islanded operation of offshore wind power plants connected through a VSC-HVDC link," in Proc. $39^{\text {th }}$ IEEE Ind. Electron. Soc. Annu. Conf., Nov. 2013, pp. 5254-5259.

\section{BIOGRAPHIES}

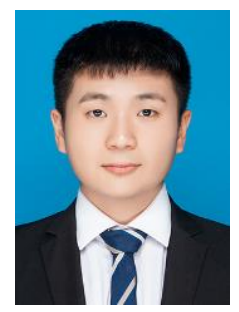

Saizhao Yang obtained his B.E. degree in electrical engineering from Huazhong University of Science and Technology (HUST), China, in 2018. He is currently pursuing his $\mathrm{PhD}$ degree at HUST. His research interests include de fault protection of MMC-HVDC and de grids.

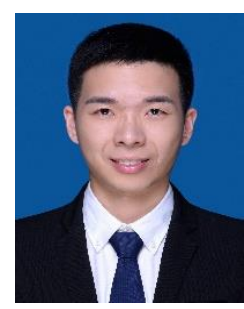

Wang Xiang (S'16-M'17) received his B.Eng. and $\mathrm{PhD}$ degrees both in electrical engineering from Huazhong University of Science and Technology (HUST), China in 2012 and 2017, respectively. He was a visiting student at the University of Aberdeen and the University of Strathclyde in 2014 and 2016 respectively. Currently, he is a research associate with the University of Strathclyde since 2018. His main research interests include MMC-HVDC, high power $\mathrm{dc} / \mathrm{dc}$ converters and dc grids.

Jinyu Wen (M'10) received his B.Eng. and Ph.D. degrees all in electrical engineering from Huazhong University of Science and Technology (HUST), Wuhan, China, in 1992 and 1998, respectively. He was a 
This paper is a post-print of a paper submitted to and accepted for publication in IEEE Transaction on Power Delivery and is subject to

visiting student from 1996 to 1997 and a research fellow from 2002 to 2003 all at the University of Liverpool, UK, and a senior visiting researcher at the University of Texas at Arlington, USA in 2010. From 1998 to 2002 he was a director engineer in XJ Electric Co. Ltd. in China. In 2003 he joined the HUST and now is a Professor at HUST. His current research interests include renewable energy integration, energy storage application, DC grid, and power system operation and control. 